\title{
ENTIRE CURVES, INTEGRAL SETS AND PRINCIPAL BUNDLES.
}

\author{
JÖRG WINKELMANN
}

\begin{abstract}
We compare the behaviour of entire curves and integral sets, in particular in relation to locally trivial fiber bundles, algebraic groups and finite ramified covers over semi-abelian varieties.
\end{abstract}

\section{SuRveY}

It has been widely conjectured by Serge Lang and other mathematicians that the diophantine behaviour of a projective variety $V$ defined over a number field $K$ is related to its complex-analytic properties. The philosophy is that for a projective variety defined over a number field $K$ infinite sets of points rational over some finite extension of $K$ should correspond to holomorphic entire curves.

For non-compact varieties there is an analoguos philosophy: Entire curves should correspond to infinite sets of integral points. (Loosily speaking integral points are points whose coordinates are integers. See discussion below.)

Conjecture 1.1. Let $X$ be an algebraic variety defined over a number field $K$.

Then there exists a non-constant holomorphic map $f: \mathbb{C} \rightarrow X(\mathbb{C})$ (with Zariski dense image) if and only if there is a finite field extension $K^{\prime} / K$ such that $X$ admits an infinite (resp. Zariski dense) integral set.

This is known to be true in dimension one: Using the uniformization theorem or Nevanlinna theory one can prove that a complex curve ("Riemann surface") $X$ admits a non-constant holomorphic map $f$ : $\mathbb{C} \rightarrow X$ if and only if $X$ is biholomorphic to $\mathbb{P}_{1}, \mathbb{C}, \mathbb{C}^{*}$ or an elliptic curve, while on the other side the theorem of Siegel (for affine curves) and Faltings proof of "Mordells conjecture" (for projective curves) imply that these are also the only algebraic curves for which the above stated arithmetic analogue holds.

It is also known to be true for subvarieties of abelian varieties. In fact, for a subvariety $Z$ of an abelian variety $A$ both properties are

1991 Mathematics Subject Classification. 14G05, 14G25, 14L10, 32H25. 
equivalent to the assumption that there is a translate $W$ of an abelian subvariety of $A$ which is contained in $Z$.

However, for arbitrary varieties in dimension two or higher this conjecture is wide open.

The purpose of this article is to provide some small steps towards this conjecture. We will discuss fiber bundles, algebraic groups and ramified coverings over semi-abelian varieties from this point of view.

We verify that integral points sets and entire curves do share some common functorial properties concerning these topics.

In particular we establish some lifting properties for fiber bundles which we use in $\S 1.4$ in a combination with a variant of Jouanolou's trick to provide a new way to define the notion of an "integral set".

1.1. Fiber bundles. If $f: \mathbb{C} \rightarrow X$ is an entire curve and $E \rightarrow X$ a holomorphic fiber bundle, then we can lift $f$ to a map $F: \mathbb{C} \rightarrow E$. We prove a strong complex-analytic statement in this direction and an arithmetic analog.

Theorem 1.2. Let $G$ be an algebraic group, $X$ an algebraic variety and $\pi: E \rightarrow X$ a G-principal bundle, all defined over a number field $K$.

Then the following assertions hold true:

a) For every holomorphic map $f: \mathbb{C} \rightarrow X(\mathbb{C})$ there exists a holomorphic $F: \mathbb{C} \rightarrow E(\mathbb{C})$ such that

(1) $f=\pi \circ F$,

(2) Assume furthermore that $G$ is connected. Then $F$ can be chosen such that the Zariski closure of the image $F(\mathbb{C})$ coincides with the preimage $\pi^{-1}\left(\overline{f(\mathbb{C})}^{\text {Zar }}\right)$ of the Zariski closure of the image of $f$.

b) Assume furthermore that the bundle $E \rightarrow X$ is locally trivial in the Zariski topology. Let $R$ be a set of integral points in $X(K)$. Then there exists a finite field extension $K^{\prime} / K$ and a set of integral points $R^{\prime} \subset E\left(K^{\prime}\right)$ such that

(1) $\pi\left(R^{\prime}\right)=R$.

(2) The Zariski closure of $R^{\prime}$ in $E$ coincides with the preimage $\pi^{-1}\left(\bar{R}^{\text {Zar }}\right)$ of the Zariski closure of $R$.

Remarks.

(1) The arithmetic statement should be true even without the additional assumption of $E \rightarrow X$ being Zariski locally trivial.

(2) No extension $K^{\prime} / K$ of the number field $K$ is needed if we keep the assumption of $E \rightarrow X$ being Zariski locally trivial and assume in addition that $G$ admits a Zariski dense integral subset. 
(3) We can not simulataneously drop the condition of being locally trivial in the Zariski topology and do without extending the field. In fact, for an arbitrary $G$-principal bundle $E \rightarrow X$ it may happen that $E(K)$ is empty even if $X(K)$ contains an integral subset dense in the Zariski topology: The finite group $\mathbb{Z} / 2 \mathbb{Z}$ is a real algebraic group as $\left\{z \in \mathbb{R}^{*}: z^{2}=1\right\}=\operatorname{Spec} \mathbb{R}[t] /\left(t^{2}-1\right)$ acting on its principal homogeneous space $E=\left\{z \in \mathbb{C}^{*}: z^{2}=\right.$ $-1\}$. Then $E(\mathbb{R})$ is empty and $E \rightarrow S p e c \mathbb{R}$ is a $G$-principal bundle.

1.2. Groups. For a complex Lie group $G$ it is easy to construct holomorphic maps $f: \mathbb{C} \rightarrow G$ due to the existence of the exponential map.

Theorem 1.3. Let $G$ be a connected complex Lie group. Then there exists a holomorphic map $f: \mathbb{C} \rightarrow G$ with dense image.

Theorem 1.4. Let $G$ be an algebraic group defined over a number field $K$.

Then there exists a finite field extension $K^{\prime} / K$ and a integral set $R \subset G\left(K^{\prime}\right)$ which is dense in the Zariski topology.

(Remark. This is of course well-known, but we may also regard this as a special case theorem 1 , if we consider $G$ as trivial $G$-principal bundle over a point.)

1.3. Ramified coverings over semi-abelian varieties. A semi-abelian variety $A$ is a group variety which admits a short exact sequence of algebraic groups

$$
1 \rightarrow T \rightarrow A \rightarrow M \rightarrow 1
$$

where $T$ is a torus and $M$ is an abelian variety.

We regard varieties admitting a finite morphism onto a semi-abelian variety.

Theorem 1.5. Let $X$ be a variety defined over a number field $K$ which admits a finite morphism $\pi$ onto a semi-abelian variety $A$.

Assume that there exists a holomorphic map $f: \mathbb{C} \rightarrow X$ which is non-constant resp. with Zariski dense image.

Then there exists a finite field extension $K^{\prime} / K$ and an integral set $R \subset X\left(K^{\prime}\right)$ such that $R$ is infinite resp. Zariski dense.

Corollary 1.6. Let $X$ be a variety defined over a number field $K$ which admits a finite morphism onto a semi-abelian variety.

Assume that every integral subset of $X\left(K^{\prime}\right)$ is finite for a every finite field extension $K^{\prime} / K$.

Then every holomorphic map from $\mathbb{C}$ to $X(\mathbb{C})$ is constant. 
1.4. Characterising integral sets. For an affine variety $V$ there is an easy definition of an "integral" set: This is a set $H$ of $K$-rational points on $V$ such that there exists a closed embedding $i$ of $V$ into an affine space $\mathbb{A}^{n}$ such that all the coordinates of all the elements $i(x)$ $(x \in H)$ are integral (i.e. in some $\mathcal{O}_{L}(S)$ where $L$ is a finite extension of $K$ and $S$ a finite set of valuations including all archimedean ones).

For arbitrary varieties there are two equivalent (see [12], proposition 1.4.7) definitions: The first definition uses models over the integers, the second Weil functions.

Here we present a third way, which we feel is more elementary.

Proposition 1.7. Let $V$ be a quasi-projective variety defined over some number field $K$. Then $H \subset V(K)$ is integral if and only if there exists a finite field extension $L / K$ and a finite set $S$ of valuations of $L$ including all archimedean ones, an affine L-variety $W$, a L-morphism $\phi: W \rightarrow$ $V$ and an integral subset $I \subset W(L)$ such that $H \subset \phi(I)$.

Proof. One direction is well-known (see [12]). The other direction follows from our Main Theorem in combination with the two lemmata below which are essentially a variant of a result known as "Jouanolou's trick", see [4].

Lemma 1.8. Let $V$ be a quasiprojective variety. Then there is a $G_{m}$ principal bundle $Q \rightarrow V$ such that $Q$ is a quasi-affine variety.

Proof. We embed $V \subset \mathbb{P}_{N}$ and take $Q=\pi^{-1}(V)$ where $\pi: \mathbb{A}^{N+1} \backslash$ $\{(0, \ldots, 0)\} \rightarrow \mathbb{P}_{N}$.

Lemma 1.9. Let $Q$ be quasi-affine variety. Then there is a principal bundle $W \rightarrow Q$ with a linear algebraic group as structure group such that $W$ is an affine variety.

Proof. We realize $Q$ as $Q=\bar{Q} \backslash Z$ where $\bar{Q}$ is an affine variety and $Z$ is a closed subvariety. Then there are regular functions $f_{1}, \ldots, f_{s}$ on $\bar{Q}$ such that $Z$ is the joint zero locus of the $f_{i}$. Then $z \mapsto\left(z, f_{1}(z), \ldots, f_{s}(z)\right)$ yields a closed embedding $i$ of $Q$ into

$$
\bar{Q} \times\left(\mathbb{A}^{s} \backslash\{(0, \ldots, 0)\}\right) .
$$

The usual action of $S L_{s}$ on $\mathbb{A}^{s}$ gives us a principal bundle $S L_{s} \rightarrow$ $\left(\mathbb{A}^{s} \backslash\{(0, \ldots, 0)\}\right)$ and thereby a principal bundle $\rho$ from $\bar{Q} \times S L_{s}$ to

$$
\bar{Q} \times\left(\mathbb{A}^{s} \backslash\{(0, \ldots, 0)\}\right) .
$$

Now we take the restriction of $\rho$ to $W=\rho^{-1}(i(Q))$. Note that $W$ is an affine variety, because it is a closed subvariety of $\bar{Q} \times S L_{s}$ and both $\bar{Q}$ and $S L_{s}$ are affine varieties. 


\section{Complex Lie Groups}

In this section we will prove the analytic part of theorem 1 .

Our first step is the auxiliary result below.

Proposition 2.1. Let $G$ be a connected complex Lie group. Then there exists a complex vector space $\mathbb{C}^{N}$ with a surjective holomorphic map $f: \mathbb{C}^{N} \rightarrow G$.

Proof. Let $\pi: \tilde{G} \rightarrow G$ be the universal covering and $\tilde{G}=S \cdot R$ its LeviMalcev decomposition, i.e., $S$ is a maximal connected semisimple Lie subgroup and $R$ is a maximal connected normal solvable subgroup. As a semisimple complex Lie group, $S$ is algebraic. Fixing a root system, we obtain a Borel subgroup $B$ corresponding to the positive roots and an "opposite Borel subgroup" $B^{-}$corresponding to the negative roots. Now $B^{-} \cdot B$ contains a Zariski open neighbourhood $V$ of $e$ in $S$. Since connected topological groups are generated as a group by each neighbourhood of the neutral element, we have $\cup_{k} V^{k}=S$. Since $S \backslash V^{k}$ is a descending sequence of closed subvarieties, it follows that $\left(B^{-} \cdot B\right)^{k}=S$ for some $k \in \mathbb{N}$. Thus there is a surjective holomorphic map from the connected solvable complex Lie group $A=\left(B^{-} \times B\right)^{k} \times R$ to $G$. This implies the statement, because for every connected solvable complex Lie group $A$ the universal covering space $\tilde{A}$ is biholomorphic to some $\mathbb{C}^{N}$.

Corollary 2.2. Let $G$ be a connected complex Lie group.

Then there exists a holomorphic map $f: \mathbb{C} \rightarrow G$ with dense image.

Proof. This follows from the theorem, because there exists a holomorphic map $f: \mathbb{C} \rightarrow \mathbb{C}^{N}$ with dense image for every $N \in \mathbb{N}$ (see e.g. [13]).

Next we need an auxiliary result.

Proposition 2.3. Let $C$ be a connected complex manifold, $\rho: C \rightarrow \mathbb{R}^{+}$ an unbounded continuous function, $X$ a complex algebraic variety and $f: C \rightarrow X$ a holomorphic map such that the image $f(C)$ is Zariski dense.

Then there exists a discrete subset $D \subset C$ such that $f(D)$ is Zariski dense in $X$. Moreover $D$ can be chosen such that $\{x \in D: \rho(x)<c\}$ is finite for every $c \in \mathbb{R}$.

Corollary 2.4. Let $C$ be a complex manifold and $F: C \rightarrow X$ be a holomorphic map to a complex algebraic variety. Then there exists a discrete subset $D \subset C$ such that the Zariski closures of $f(C)$ and $f(D)$ coincide. 
Proof. Let $\mathcal{I}$ be the set of all discrete subsets $D \subset C$ for which

(1) all the sets $\{x \in D: \rho(x)<c\}$ are finite and

(2) the Zariski closure of $f(D)$ in $X$ is irreducible.

Then we define $\mathcal{M} \subset \mathcal{I}$ as the family of all those $D \in \mathcal{I}$ which are maximal in the following sense: If $D^{\prime} \in \mathcal{I}$ with $\overline{f(D)}^{\text {Zar }} \subset{\overline{f\left(D^{\prime}\right)}}^{\text {Zar }}$, then $\overline{f(D)}^{\text {Zar }}={\overline{f\left(D^{\prime}\right)}}^{\text {Zar }}$.

We claim that there are only finitely many subvarieties $V \subset X$ arising as the Zariski closure of an image $f(D)$ for some $D \in \mathcal{M}$. Indeed, let us assume the converse and let $D_{n}$ be an infinite sequence in $\mathcal{M}$ for which the subvarieties ${\overline{f\left(D_{n}\right)}}^{Z a r}$ are all different. Then we define $\Delta \subset C$ via

$$
\Delta=\cup_{n \in \mathbb{N}}\left\{x \in D_{n}: \rho(x)>n\right\}
$$

By construction $\{x \in \Delta: \rho(x)<c\}$ is finite for every $c \in \mathbb{R}$. This implies that $\Delta$ is discrete. For each $n \in \mathbb{N}$ the set $\Delta$ contains all but finitely many elements of $D_{n}$. Since ${\overline{f\left(D_{n}\right)}}^{\text {Zar }}$ is irreducible, it follows that

$$
{\overline{f\left(D_{n}\right)}}^{Z a r}={\overline{f\left(D_{n} \cap \Delta\right)}}^{Z a r} \subset \overline{f(\Delta)}^{\text {Zar }}
$$

for each $n \in \mathbb{N}$. Now let $Z$ be an irreducible component of $\overline{f(\Delta)}^{\text {Zar }}$. Then $Z \cap \Delta \in \mathcal{I}$. By the maximality assumption for the $D_{n}$ it follows that ${\overline{f\left(D_{n}\right)}}^{Z a r}$ is an irreducible component of $\overline{f(\Delta)}^{Z a r}$ for each $n \in$ $\mathbb{N}$. Since $\overline{f(\Delta)}{ }^{Z a r}$ has only finitely many irreducible components, we deduce that there exist only finitely many subvarieties $V \subset X$ realizable as $V=\overline{f(D)}^{Z a r}$ for some $D \in \mathcal{M}$.

Now let $Z_{0}$ be the union of all such $V$. Since there are only finitely many such $V$, the union $Z_{0}$ is a subvariety.

Next we claim that $Z_{0}=X$. Indeed, if not, then $f^{-1}\left(X \backslash Z_{0}\right)$ is a dense open subset of $C$ which allows as to choose an infinite subset $D^{\prime} \subset C$ such that $f\left(D^{\prime}\right) \cap Z_{0}=\{\}$ and such that $\left\{x \in D^{\prime}: \rho(x)<c\right\}$ is finite for every $c \in \mathbb{R}$. Now for every irreducible component $W$ of ${\overline{f\left(D^{\prime}\right)}}^{Z a r}$ we obtain an element $W \cap D^{\prime} \in \mathcal{I}$. By noetherianity there must exist an element $D \in \mathcal{M}$ with ${\overline{f\left(W \cap D^{\prime}\right)}}^{Z a r} \subset \overline{f(D)}^{\text {Zar }}$ which leads to a contradiction, because $f\left(D^{\prime}\right)$ does not intersect $Z_{0}$. Thus $Z_{0}=X$. Since $X=\overline{f(C)}^{Z a r}$ is irreducible, it follows that $X=\overline{f(D)}^{\text {Zar }}$ for some $D \in \mathcal{M}$.

Theorem 2.5. Let $G$ be a complex algebraic group and $\pi: E \rightarrow X$ be a complex algebraic G-principal bundle (locally trivial in the étale topology). 
Then for every holomorphic map $f: \mathbb{C} \rightarrow X(\mathbb{C})$ there exists a holomorphic map $F: \mathbb{C} \rightarrow E(\mathbb{C})$ such that

(1) $f=\pi \circ F$,

(2) The Zariski closure of the image $F(\mathbb{C})$ coincides with the preimage $\pi^{-1}\left(\overline{f(\mathbb{C})}^{\text {Zar }}\right)$ of the Zariski closure of the image of $f$.

Proof. There is no loss in generality in assuming that $f(\mathbb{C})$ is Zariski dense in $X$.

The pull back bundle $E \times_{X} \mathbb{C} \rightarrow \mathbb{C}$ is a holomorphic $G$-principal bundle over $\mathbb{C}$. It is topologically trivial, because $\mathbb{C}$ is a contractible topological space. Due to Grauert's Oka principle ([3]) this implies that it is holomorphically trivial and therefore admits a holomorphic section $\sigma: \mathbb{C}$ to $E \times{ }_{X} \mathbb{C}$.

On the other hand, $X$ contains a Zariski open subset $U$ which admits an étale cover $V \rightarrow U$ such that the pull-back bundle of $E$ is trivial over $V$. Define $U^{\prime}=f^{-1}(U)$ and $V^{\prime}=V \times_{U} U^{\prime}$. Now we can choose two trivializations of the pull-back bundle over $V^{\prime}$ : First, we choose a holomorphic section $\sigma: \mathbb{C} \rightarrow E \times_{X} \mathbb{C}$ which yields a holomorphic section $\sigma^{\prime}$ of $E^{\prime}=E \times_{X} V^{\prime} \rightarrow V^{\prime}$. Second, the assumption that $E \times_{X}$ $V \rightarrow V$ is algebraically trivial permits us to chose an algebraic section $\eta$ of $E \times_{X} V \rightarrow V$ which induces a holomorphic section $\eta^{\prime}$ of $E^{\prime}=$ $E \times_{X} V \rightarrow V^{\prime} \rightarrow V^{\prime}$.

Then there exists a holomorphic map $\zeta: V^{\prime} \rightarrow G$ such that

$$
\sigma^{\prime}(x)=\eta^{\prime}(x) \cdot \zeta(x) \forall x \in V^{\prime}
$$

where $\cdot$ denotes the $G$-principal right action on $E^{\prime}=E \times_{X} V^{\prime}$.

There is a unbounded continuous function $\rho: V^{\prime} \rightarrow \mathbb{R}$ given by $\rho(x)=|\tau(x)|$.

We claim that there exists sequences of discrete subsets $D_{n} \subset V^{\prime}$ with the following properties:

- For each $n \in \mathbb{N}$ the projection map $\left.\tau\right|_{D_{n}}: D_{n} \rightarrow \tau\left(D_{n}\right)$ is injective.

- All the sets $\tau\left(D_{n}\right)$ are disjoint and their union is discrete in $\mathbb{C}$.

- For each $D_{n}$ the image in $V$ is Zariski-dense under the natural $\operatorname{map} V^{\prime}=V \times_{B} \mathbb{C} \rightarrow V$.

We choose the subsets $D_{n}$ recursively. Suppose $D_{1}, \ldots, D_{n-1}$ already chosen. Let $C=V^{\prime} \backslash \cup_{k<n} \tau^{-1}\left(\tau\left(D_{k}\right)\right)$ and $X=V$ and use proposition 2.3. Since $\tau: V^{\prime} \rightarrow \mathbb{C}$ has finite fibers, we can decompose the subset $D$ thus obtained as a finite union of $D=\Delta_{1} \cup \ldots \Delta_{r}$ such that no fiber $\tau^{-1}(x)(x \in \mathbb{C})$ intersects any $\Delta_{i}$ in more than one point. The union of the Zariski closures of the images of the $\Delta_{i}$ in $V$ equals tha Zariski closure of the image of $D$ which is $V$. Since 
$V$ is irreducible, we deduce that we can choose one $\Delta_{i}$ for which the image in $V$ is Zariski dense. We fix this index $i$ and choose $D_{n}$ as $D_{n}=\left\{x \in \Delta_{i}: \rho(x)=|\tau(x)|>n\right\}$.

Chosen in this way, the sequence of subsets $D_{n}$ has the desired properties.

Next we fix a countable dense subset $\Sigma \subset G$ and a bijection $\alpha$ : $\mathbb{N} \rightarrow \Sigma$. There is surjective holomorphic map $\xi$ from some vector space $\mathbb{C}^{N}$ onto $G$ (theorem 2.1). We choose elements $v_{n} \in \mathbb{C}^{N}$ such that $\xi\left(v_{n}\right)=\alpha(n)$ for all $n \in \mathbb{N}$.

Define $\Delta=\cup_{n} \tau\left(D_{n}\right) \subset \mathbb{C}$. By construction $\Delta$ is discrete in $\mathbb{C}$. We can thus chose a holomorphic function $\Phi: \mathbb{C} \rightarrow \mathbb{C}^{N}$ with $\Phi(\tau(x))=v_{n}$ for all $n \in \mathbb{N}$ and all $x \in D_{n}$. Define $\beta=\xi \circ \Phi: \mathbb{C} \rightarrow G$. Then $\beta(\tau(x))=\alpha(n)$ for all $x \in D_{n}$.

Again using the surjective map $\xi: \mathbb{C}^{N} \rightarrow G$, we may chose a holomorphic function $\gamma: \mathbb{C} \rightarrow G$ with $\gamma(\tau(x))=\zeta(x)$ for all $n \in \mathbb{N}$ and all $x \in D_{n}$.

Next we define a new section $\tilde{\sigma}$ in the pull-back bundle $E \times_{X} \mathbb{C} \rightarrow \mathbb{C}$ as follows:

$$
\tilde{\sigma}(z)=\sigma(z) \cdot\left(\gamma(z)^{-1}\right) \cdot \beta(z) \quad(z \in \mathbb{C})
$$

where denote the right $G$-action on the principal bundle.

Then

$$
\begin{aligned}
\tilde{\sigma}(\tau(x)) & =\sigma(\tau(x)) \cdot\left(\zeta(x)^{-1}\right) \cdot \beta(\tau(x)) \\
& =\eta^{\prime}(x) \cdot \beta(\tau(x))
\end{aligned}
$$

for all $x \in V^{\prime}$ and therefore

$$
\tilde{\sigma}(\tau(x))=\eta^{\prime}(x) \cdot \alpha(n)
$$

for all $x \in D_{n}$ (and all $\left.n \in \mathbb{N}\right)$.

Let $Z=\eta(V) \subset E_{X} V$. This is an algebraic subvariety, since $\eta$ is algebraic. Let $\epsilon$ denote the natural map from $E^{\prime}=E_{X} V^{\prime}$ to $E \times_{X} V$ and let $\chi$ denote the natural map from $V^{\prime}$ to $V$. Then

$$
\epsilon\left(\tilde{(} \sigma\left(\left(\tau\left(D_{n}\right)\right)\right)=\epsilon\left(\eta\left(D_{n}\right)\right) \cdot \alpha(n)=\eta\left(\chi\left(D_{n}\right)\right) \cdot \alpha(n)\right.
$$

is Zariski dense in $Z \cdot \alpha(n)$. As a consequence, the (algebraic) Zariski closure of $\cup_{n} \epsilon\left(\tilde{\sigma}\left(\tau\left(D_{n}\right)\right)\right)$ contains all $Z \cdot \alpha(n)(n \in \mathbb{N})$. This implies that $\epsilon\left(\tilde{\sigma}\left(\tau\left(V^{\prime}\right)\right)\right)$ is Zariski dense in $E^{\prime}$. Therefore $\epsilon(\tilde{\sigma}(\mathbb{C}))$ induces a holomorphic map from $\mathbb{C}$ to $E$ with Zariski dense image. Hence the assertion.

Proposition 2.6. Let $C$ be a Stein complex manifold, let $X$ be a complex algebraic variety on which a connected complex Lie group $G$ acts and let $f: C \rightarrow X$ be a holomorphic map. 
Then there exists a holomorphic map $\alpha: C \rightarrow G$ such that the map $F: C \rightarrow X$ defined by $F(x)=f(x) \cdot \alpha(x)$ fulfills the following property:

The (algebraic) Zariski closure of $F(C)$ is $G$-invariant and contains the Zariski closure of $f(C)$.

Proof. For each holomorphic map $\alpha: C \rightarrow G$ let $F_{\alpha}: C \rightarrow X$ denote the map defined by $F_{\alpha}(x)=f(x) \alpha(x)$. Among all these maps $F_{\alpha}$ we choose one for which the dimension of the Zariski closure of the image $F_{\alpha}(C)$ in $X$ is maximal. We claim that this $F_{\alpha}$ has the desired property.

Let $D \subset C$ be a discrete subset for which the Zariski closures of $f(C)$ and $f(D)$ coincide. Now assume that the Zariski closure $A$ of $F_{\alpha}(C)$ is not $G$-invariant. Then there exists a point $p \in C \backslash D$ such that $A$ does not contain the $G$-orbit through $F_{\alpha}(p)$. Fix such a point $P$ and an element $g \in G$ for which $p \cdot g \notin A$. Next we choose a holomorphic map $\beta: C \rightarrow G$ such that $\beta(x)=\alpha(x)$ for every $x \in D$ and such that $\beta(p)=\alpha(p) \cdot g$. Then the Zariski closure of $F_{\beta}(C)$ contains $F_{\alpha}(D)=F_{\beta}(D)$. By the choice of $D$ it follows that the Zariski closure of $F_{\beta}(C)$ contains that of $F_{\alpha}(C)$. By the maximality condition and the fact that $C$ is an irreducible space it follows that the Zariski closure of $F_{\alpha}(C)$ and $F_{\beta}(C)$ coincide. This is a contradiction, because $p \cdot g$ is contained in $F_{\beta}(C)$, but not in the Zariski closure of $F_{\alpha}(C)$. Hence $\overline{F_{\alpha}(C)}$ needs to be $G$-invariant.

\section{INTEGRAL SETS}

We use the notation of Lang and Vojta.

Let $X$ be a projective variety defined over a number field $K$ with algebraic closure $\bar{K}, M$ the set of all places of $K, S$ a set of places containing all the archimedean ones, and $D$ a Cartier divisor on $X$ defined over $K$. In this situation Lang defined $([5])$ the notion of a "Weil function" $\lambda_{D}: M \times X(\bar{K}) \rightarrow \mathbb{R}$.

A collection of real numbers $C_{v}$ (with $v \in M$ ) is called an " $M$ constant" if $C_{v}=0$ for all but finitely many $v \in M$.

If $D$ is very ample and $i: X \backslash|D| \hookrightarrow \mathbb{A}^{N}$ a closed embedding given by the sections of $D$, then a possible choice for a Weil function is

$$
\lambda_{D}(v, x)=\log ^{+} \max _{k=1 \ldots N}\left|(i(x))_{k}\right|_{v}
$$

where $\log ^{+}(w)$ is defined as $\max \{0, w\}$.

A set $A \subset X(K)$ is called " $(S, D)$-integral" if there is an $M$-constant $C_{v}$ such that $\lambda_{D}(p, v) \leq C_{v}$ for all $p \in A$ and $v \notin S$.

3.1. Non-compact varieties. Let $X$ be a non-complete variety. By a result of Nagata [6] $X$ can be embedded into a complete variety $\bar{X}$. 
Due to the desingularization theorems in characteristic zero we may assume that $\bar{X}$ is smooth and $\bar{X} \backslash X$ is a s.n.c. divisor provided $X$ is smooth.

Definition 3.1. Let $X$ be a (non-complete) smooth variety.

A function $\mu: X(\bar{k}) \times M \rightarrow \mathbb{R}$ is called "Weil function for $X$ " if there exists a completion $X \hookrightarrow \bar{X}$ by a divisor $D=\bar{X} \backslash X$ and constants $C>1, C^{\prime}>0$ such that

$$
C \lambda_{D}(x, v)+C^{\prime} \geq \mu(x, v) \geq \frac{1}{C} \lambda_{D}(x, v)-C^{\prime}
$$

for all $x \in X(\bar{k})$.

Definition 3.2. A subset $A \subset X(k)$ is called an "S-integral set" iff there is a Weil function $\lambda$ for $X$ such that $\lambda(x, v) \leq 0$ for all $x \in A$, $v \in M_{k} \backslash S$.

$A$ is called an "integral set" if there exists a finite subset $S \subset M_{k}$ which includes all archimedean places for which $A$ is an $S$-integral set.

Equivalently: A subset $A \subset X(k)$ is an integral set if there exists a compactification $X \hookrightarrow \bar{X}$ such that $D=\bar{X} \backslash X$ is a Cartier divisor and $A$ is an $(S, D)$-subset of $\bar{X}$.

Lemma 3.3. Let $X$ be a variety. If $\lambda$ and $\tilde{\lambda}$ are two Weil functions for $X$, then there is a real number $C \geq 1$ and $M$-constants $a_{v}$ such that $\lambda_{v} \leq a_{v}+C \tilde{\lambda}_{v}$.

Proof. If $X \hookrightarrow \bar{X}$ and $X \hookrightarrow \tilde{X}$ are two completions by divisors, we may consider the diagonal embedding $X \hookrightarrow \bar{X} \times \tilde{X}$. Let $Y$ denote the closure of $X$ in $\bar{X} \times \tilde{X}$. Let $D=\bar{X} \backslash X$ and $\tilde{D}=\tilde{X} \backslash X$. Then $p_{1}^{*} D$ and $p_{2}^{*} \tilde{D}$ have the same support in $Y$. Hence the statement.

\subsection{Examples.}

(1) If $V$ is projective, any set of $K$-rational points is integral.

(2) If $V$ is affine, a subset $A \subset V(K)$ is $S$-integral iff there exists a proper embedding $i \hookrightarrow \mathbb{A}^{N}$ with $i(A) \subset \mathbb{A}^{N}\left(\mathcal{O}_{S}\right)$.

(3) If $V=\mathbb{A}^{n} \backslash\{(0, \ldots, 0)\}$ an $S$-integral set is given by taking all $\left(x_{1}, \ldots, x_{n}\right) \in \mathcal{O}_{S}^{n}$ such that $x_{1}, \ldots, x_{n}$ generate the unit ideal of $\mathcal{O}_{S}$.

(4) If $V=\mathbb{P}^{n} \backslash\{[1: 0: \ldots: 0]\}$ an $S$-integral set is given by taking all $\left[x_{0}: \ldots: x_{n}\right]$ such that $x_{0}$ is contained in $x_{1} \mathcal{O}_{S}+\ldots+x_{n} \mathcal{O}_{S}$.

(5) If $V=G_{m}$ (the multiplicative group), the subgroup of units $\mathcal{O}_{S}^{*}$ is an $S$-integral set.

(6) If $A$ is an $S$-integral set in a variety $V$ which admits a morphism $f$ to a variety $W$, then $f(A)$ is a $S$-integral set. 
(7) Let $\pi: V \rightarrow W$ be an étale covering, defined over some number field $K$ and $A$ a $S$-integral subset of $W$. Then there is a finite field extension $F / K$ such that $\pi^{-1}(A) \subset V(F)$. Moreover $\pi^{-1}(A)$ is a $T$-integral set where $T$ denotes the set of all non-archimedean places of $F$ lying over $S$.

(8) Every finite subset is an integral set.

(9) If $A$ is a $S$-integral subset of a variety $W$ and $f: V \rightarrow W$ is a proper morphism, all defined over a number field $K$, then $f^{-1}(A) \cap V(K)$ is a $S$-integral set.

\section{Fibrations}

In real geometry, if $G$ is a Lie group and $H \subset I \subset G$ are closed Lie subgroups then the fibration $G / H \rightarrow G / I$ is "locally trivial" in the ordinary topology. In algebraic geometry, if $G$ is an algebraic group and $H \subset I \subset G$ are algebraic subgroups, then $G / H \rightarrow G / I$ is not necessarily locally trivial with respect to the Zariski topology, but always locally trivial with respect to étale topology as already observed in [10]. Following [10], an algebraic group is called "special" if such a fibration is necessarily locally trivial for the Zariski topology. A nontrivial finite group can not be special: For every such group $G$ there is a unramified Galois covering $X^{\prime} \rightarrow X$ of complex algebraic curves with $G$ as Galois group. This is a $G$-principal bundle which is evidently not locally trivial in the Zariski topology.

On the other hand, all solvable connected algebraic groups as well as certain semisimple groups like e.g. $S L_{n}$ are special. However, there are simple connected algebraic groups which are not special, e.g. $S O_{n}$ $([10])$.

From now on a $G$-principal bundle $\pi: E \rightarrow B$ is a principal bundle which is locally trivial with respect to the étale topology. In other words, there is a free right action of $G$ on $B$ such that the $G$-orbits are the fibers of $\pi$ and for every point $p \in B$ there is a Zariski open neighbourhood $U$ and an étale covering $\tau: V \rightarrow U$ such that there is a $G$-equivariant isomorphism between $V \times{ }_{B} E$ and $V \times G$. If $p \in B(k)$, then $\pi^{-1}(p)$ is a $G$-principal homogeneous space. If there is a $k$-rational point $q \in V(k)$ with $\tau(q)=p$, this principal homogeneous space is isomorphic to $G$, i.e., it contains $k$-rational points. However, in general for an étale morphism $\tau$ one can not find a $k$-rational point in $V$ over each $k$-rational point in $U$. For an easy example of this phenomenon consider $\tau: V \rightarrow U$ given by $V=U=\mathbb{A} \backslash\{0\}$ and $\tau(z)=z^{2}$. 


\section{Restricted topological PRODUCT}

Let us recall the theory of restricted topological products.

If $\left(X_{\lambda}\right)$ is a family of locally compact topological spaces, each endowed with a compact open subspace $Y_{\lambda}$, then one can define a "restricted topological product". The idea is to modify the direct product of all $X_{\lambda}$ in such a way that the resulting space is locally compact. (In general an infinite product of locally compact spaces is not locally compact.) As a set, the "restricted topological product" contains all

$$
x=\left(x_{\lambda}\right) \in \Pi_{\lambda} X_{\lambda}
$$

such that $x_{\lambda} \notin Y_{\lambda}$ for only finitely many $\lambda$. Thus, $X=\cup_{S} X(S)$ where $S$ runs through all finite subsets of the index set and $X(S)=$ $\left(\Pi_{\lambda \in S} X_{\lambda}\right) \times\left(\Pi_{\lambda \notin S} Y_{\lambda}\right)$. Now a locally compact topology is introduced on $X$ as follows: A subset $U \subset X$ is open if and only if $U \cap X(S)$ is open in $X(S)$ (with respect to the product topology on $X(S)$ ) for all $S$. (Warning: This is not the topology obtained by embedded $X$ into the direct product of all $X_{\lambda}$.)

Lemma 5.1. Let $X$ be as above and let $\rho_{\lambda}: X_{\lambda} \rightarrow \mathbb{R}$ be a collection of continuous functions such that $\rho_{\lambda}$ vanishes in $Y_{\lambda}$ for almost all $\lambda$.

Then

$$
\rho(x)=\sum_{\lambda} \rho_{\lambda}\left(x_{\lambda}\right)
$$

defines a continuous function on $X$.

Proof. First we note that for each fixed $x \in X$ the sum on the right is actually finite, thus $\rho: X \rightarrow \mathbb{R}$ is well-defined. Let $T$ be the set of all $\lambda$ for which $\rho_{\lambda}$ does not vanish on $Y_{\lambda}$. Then

$$
\rho(x)=\sum_{\lambda \in S \cup T} \rho_{\lambda}\left(x_{\lambda}\right)
$$

for every finite index set $S$ and every $x \in X(S)$. This being a finite sum implies that the restriction of $\rho$ to each $X(S)$ is continuous. Therefore $\rho^{-1}(W) \cap X(S)$ is open for every $S$ and every open subset $W \subset \mathbb{R}$. Hence $\rho$ is continuous.

\section{Adelic groups}

Let $K$ be a number field, $M$ the set of absolute values, $M_{\infty}$ the set of archimedean absolute values and $M_{f}$ the set of non-archimedean ones. As usual, for each $v \in M$ we denote by $K_{v}$ the completion of $K$ with respect to the absolute value $v$.

Then the ring of Adeles $\mathbf{A}$ is defined as the restricted topological product of all $K_{v}$ where the role of the compact open subsets $Y_{\lambda}$ is 
played by the ring of $v$-integers $\mathcal{O}_{v}=\left\{x \in K_{v}:|x|_{v} \leq 1\right\}$ if $v$ is non-archimedean and the empty set if $v$ is archimedean.

The ring $\mathbf{A}_{f}$ of "finite Adeles" is the restricted topological product for only the non-archimedean valuations.

It is easy to verify that $\mathbb{A}$ and $\mathbf{A}_{f}$ are topological rings in a natural way.

6.1. Adelic groups. Let $G$ be a linear $K$-group. Then $G$ is defined as an algebraic subgroup of $G L_{n}$ as the zero-set of certain polynomials with coefficients in $K$. For each $v \in M$ we define $G\left(K_{v}\right)$ and for each $v \in M_{f}$ a compact open subgroup $G\left(O_{v}\right)=G\left(K_{v}\right) \cap G L_{n}\left(O_{v}\right)$ where $O_{v}$ is the ring of integers of $K_{v}$, i.e. $O_{v}=\left\{x \in K_{v}:\|x\|_{v} \leq 1\right\}$. As usual, $G L_{n}\left(O_{v}\right)$ denotes the subgroup of all matrices $A$ in $G L_{n}\left(K_{v}\right)$ such that all matrix coefficients and $1 / \operatorname{det}(A)$ are in $O_{v}$.

The adelic group is the restricted topological product of all these groups and will by denoted by $G(\mathbb{A})$ (resp. $G\left(\mathbf{A}_{f}\right)$ if we consider the restricted topological product with respect to only the non-archimedean absolute values of $K$ ).

6.2. A finiteness theorem of Borel. A finiteness theorem of Borel has the following consequence which we will need later on.

Proposition 6.1. Let $G$ be a linear algebraic group defined over some number field $K, G\left(\mathbf{A}_{f}\right)$ the finite adelic group and $G(K)$ the group of $K$-rational points, embedded diagonally into $G\left(\mathbf{A}_{f}\right)$.

Then there exists a compact subset $C \subset G\left(\mathbf{A}_{f}\right)$ such that $C \cdot G(K)=$ $G\left(\mathbf{A}_{f}\right)$.

Proof. The locally compact group $G\left(\mathbf{A}_{f}\right)$ contains $H=\Pi_{v} G\left(\mathcal{O}_{v}\right)$ as a compact open subgroup. By a theorem of Borel ([1]) the double quotient $H \backslash G\left(\mathbf{A}_{f}\right) / G(K)$ is finite. Hence there is a finite subset $E \subset$ $G\left(\mathbf{A}_{f}\right)$ such that $G\left(\mathbf{A}_{f}\right)=H \cdot E \cdot G(K)$. Evidently $C=H \cdot E$ is compact.

\section{WEIL FUNCTIONS FOR FIBER BUNDLES}

Here we relate Weil functions on fiber bundles to Weil functions on base and fiber of such a bundle.

Proposition 7.1. Let $\pi: E \rightarrow B$ be a Zariski locally trivial fiber bundle. Let $\left(U_{i}\right)_{i \in I}$ be a family of Zariski open subvarieties of $B$ which cover all of $B$ and such that $W_{i}=\pi^{-1}\left(U_{i}\right) \rightarrow U_{i}$ is trivial for every $i \in I$, i.e., there are morphisms $\zeta_{i}: W_{i} \rightarrow F$ such that there is an isomorphism $W_{i} \rightarrow U_{i} \times F$ given by $x \mapsto\left(\pi(x), \zeta_{i}(x)\right)$. Assume all defined over some number field $K$. 
Assume the index set I to be totally ordered.

Let $\lambda_{B}, \lambda_{F}$ be a Weil functions for $B$ resp. $F$ and define $\mu$ as follows:

$$
\mu(x, v)=\lambda_{F, v} \circ \zeta_{i}(x)
$$

if for all $j \in I$ either $\lambda_{U_{j}, v}(\pi(x))>\lambda_{U_{i}, v}(\pi(x))$ or $j \geq i$ and $\lambda_{U_{j}, v}(\pi(x))=$ $\lambda_{U_{i}, v}(\pi(x))$.

Then there is a global Weil function $\lambda_{E}$ for $E$ such that $\lambda_{E} \leq \mu+$ $\lambda_{B} \circ \pi$.

Proof. For each index $i$ a Weil function for $W_{i}$ is given by

$$
\lambda_{W_{i}}=\lambda_{U_{i}} \circ \pi+\lambda_{F} \circ \zeta_{i} .
$$

Since $E$ is covered by the open subvarieties $W_{i}$, a Weil function for $E$ can be defined by

$$
\lambda_{E}=\inf _{i} \lambda_{W_{i}}=\inf _{i}\left(\lambda_{U_{i}} \circ \pi+\lambda_{F} \circ \zeta_{i}\right) .
$$

It follows that

$$
\lambda_{E, v}(x) \leq\left(\inf _{i}\left(\lambda_{U_{i}, v} \circ \pi(x)\right)+\lambda_{F, v} \circ \zeta_{j}(x) .\right.
$$

for all $v, j$ and $x \in W_{j}$. Therefore $\lambda_{E} \leq \mu+\lambda_{B} \circ \pi$.

\section{Weil FUnCtions AND Adeles}

Proposition 8.1. Let $G$ be an algebraic group defined over a number field $K$ and let $E \rightarrow B$ be a Zariski locally trivial $G$-principal bundle, likewise defined over $K$. Let $\lambda_{B}$ be a Weil function for $B$.

Then there exists a Weil function $\lambda_{E}$ for $E$ such that for every $x \in$ $B(K)$ there exists a point $y \in E(K)$ with $\lambda_{B}(x) \leq \lambda_{E}(x)$, i.e.,

$$
\lambda_{E, v}(y) \leq \lambda_{B, v}(x) \quad \forall v \in M .
$$

Proof. Consider a closed embedding $i: G \rightarrow G L_{n}$. The matrix coefficients of this embedding together with $\operatorname{det}()^{-1}$ yield an embedding $\alpha$ of $G$ as a closed subvariety into $\mathbb{A}^{n^{2}+1}$. Now we can choose a global Weil function $\lambda_{G}$ for $G$ via

$$
\lambda_{G}(v, x)=\lambda_{G, v}(x)=\log ^{+} \max \left\{\left|\alpha_{k}(x)\right|_{v}: 1 \leq k \leq n^{2}+1\right\}
$$

where $\log ^{+}(w)=\max \{0, w\}$. Each $\lambda_{G, v}$ extends to a function defined on $G\left(K_{v}\right)$ where $K_{v}$ denotes the completion of $K$ with respect to $v$. By lemma 5.1 we obtain a continuous function $\eta$ on the adelic group $G\left(\mathbf{A}_{f}\right)$ via

$$
\eta(x)=\sum_{v} \lambda_{G, v}\left(x_{v}\right)
$$

Note that

$$
\eta(x) \geq \max _{v} \lambda_{G, v}\left(x_{v}\right)
$$


because $\lambda_{G, v}\left(x_{v}\right) \geq 0$.

By proposition 6.1 there is a compact subset $C$ of $G\left(\mathbf{A}_{f}\right)$ such that $C \cdot G(K)=G\left(\mathbf{A}_{f}\right)$. Let $c^{\prime}=\max \{\eta(x): x \in C\}$. Let $d=\operatorname{deg}(K / \mathbb{Q})$ and define $S$ as the set of all $v \in M_{K}$ such that $\frac{1}{d} \log p \leq c^{\prime}$ where $p$ is the rational prime with $p \mid v$. Note that $S$ is a finite set. Hence we obtain a $M$-constant $c_{v}$ by defining

$$
c_{v}= \begin{cases}c^{\prime} & \text { if } v \in S \\ 0 & \text { else } .\end{cases}
$$

By the construction of $\lambda_{G}$ we know: If there exists an element $x \in G(K)$ and a place $v \in M_{K}$ with

$$
0<\lambda_{G, v}(x) \leq c^{\prime},
$$

then there exists a number $z \in K$ with

$$
0<\log |z|_{v} \leq c^{\prime}
$$

which in turn implies $v \in S$. It follows that for $v \notin S$ and $x \in G(K)$ the conditions " $\lambda_{G, v}(x) \leq c$ " and " $\lambda_{G, v}(x) \leq 0$ " are equivalent. Therefore $\eta(x) \leq c^{\prime}$ for $x \in G(K)$ implies

$$
\lambda_{G, v}(x) \leq c_{v} \forall v \in M
$$

Since $G(K)$ is dense in $G\left(K_{v}\right)$ for every $v$, the same conclusion holds for every $x \in G\left(\mathbf{A}_{f}\right)$.

Let $\mu$ be defined as in proposition [7.1. Proposition 7.1 states that there is a Weil function $\hat{\lambda}_{E}$ for $E$ such that $\hat{\lambda}_{E} \leq \mu+\lambda_{B} \circ \pi$. We define $\lambda_{E, v}=\hat{\lambda}_{E, v}-c_{v}$. Recall that the sum of a Weil function and an $M$-constant ist still a Weil function. Thus we found a Weil function $\lambda_{E}$ for $E$ with the property

$$
\lambda_{E, v}+c_{v} \leq \mu_{v}+\lambda_{B, v} \circ \pi \forall v .
$$

Now we fix a point $x \in B(K)$ and choose a point $p \in E(K)$ with $\pi(p)=x$. Let $\left(U_{i}\right)$ be a family of Zariski open subsets of $B$ as in proposition 7.1 and let $U_{0}, \ldots U_{r}$ denote those of the open sets $U_{i}$ which contain $x$. Let $\tau_{i}: U_{i} \rightarrow G$ be defined as in proposition 7.1 . Then there are elements $g_{0}, \ldots, g_{r} \in G$ with

$$
\tau_{i}(q)=\tau_{0}(q) \cdot g_{i}
$$

for all $0 \leq i \leq r$ and $q \in E(K)$ with $\pi(q)=x$.

There is a map $\xi: M_{K} \rightarrow\{0, \ldots, r\}$ such that

$$
\mu(q, v)=\lambda_{G, v}\left(\tau_{\xi(v)}(q)\right) . \forall q \in \pi^{-1}(x) \cap E(K) .
$$


We define an element $a=\left(a_{v}\right)_{v}$ in the finite adelic group $G\left(\mathbf{A}_{f}\right)$ as follows: $a_{v}=g_{\xi(v)} \cdot \tau_{0}(p)=\tau_{\xi(v)}(p)$. Then

$$
\mu_{v}(p \cdot g)=\lambda_{G, v}\left(\tau_{\xi(v)}(p \cdot g)\right)=\lambda_{G, v}\left(a_{v} \cdot g\right) \forall g \in G(K) .
$$

Next we choose $g \in G(K)$ such that $a \cdot g \in C$ and define $y=p \cdot g$. Then $\eta(a \cdot g) \leq c^{\prime}$ which in turn implies

$$
\mu_{v}(y)=\mu_{v}(p \cdot g)=\lambda_{G, v}\left(a_{v} \cdot g\right) \leq c_{v} \forall v \in M_{K}
$$

It follows that

$$
\lambda_{E, v}(y) \leq \lambda_{B, v}(\pi(y))+\mu_{v}(y)-c_{v} \leq \lambda_{B, v}(\pi(y))=\lambda_{B, v}(x)
$$

as desired.

Corollary 8.2. Let $G$ be an algebraic group and let $\pi: E \rightarrow B$ be a Zariski locally trivial $G$-principal bundle, all defined over some number field $K$. Let $S$ be a finite set of places of $K$.

Let $A$ be a S-integral subset of $B(K)$ and assume that $E(K) \neq \emptyset$.

Then there exists a $S$-integral subset $A^{\prime} \subset E(K)$ with $\pi\left(A^{\prime}\right)=A$.

Proof. By assumption there is a $G$-principal homogeneous space $F$ such that every point $p \in B(K)$ admits a Zariski open neighbourhood $U$ for which $\pi^{-1}(U)(K) \simeq U(K) \times F(K)$. Therefore the condition $E(K) \neq \emptyset$ implies $F(K) \neq \emptyset$ which in turn implies that $\pi(E(K))=B(K)$.

Hence the assertion.

Proposition 8.3. Let $G$ be an algebraic group defined over a number field $K$.

Then there exists a finite field extension $L / K$ such that $G(L)$ is Zariski dense in $G$.

Proof. As usual, let $\bar{K}$ denote an algebraic closure of $K$. Let us consider all algebraic subgroups which arise as the Zariski connected component of a Zariski closure of a finitely generated subgroup of $G(\bar{K})$. Let $H$ be maximal among these algebraic subgroups. One verifies easily that $H$ is normal and that every element in $(G / H)(\bar{K})$ is of finite order. This implies that $G / H$ contains no algebraic subgroup isomorphic to the additive group $G_{a}$ or the multiplicative group $G_{m}$. As a consequence, the connected component $A$ of $G / H$ must be an abelian variety. Unless $A$ is finite, it contains, like every projective variety, $\bar{K}$-rational points of non-zero height. However, as an abelian variety, $A$ admits a canonical height, the Neron-Tate height, which is zero for every torsion element. Therefore $A(\bar{K})$ must contain an element of infinite order unless $\operatorname{dim} A=0$. Thus $G / H$ must be finite. Now we can chose $L$ such that it contains the fields of definition for one point in each connected component of $G$ and for each element in a finite set of generators for 
some subgroup whose Zariski closure contains $H$. Then $G(L)$ is Zariski dense in $G$.

Remark 8.4. If $G$ is linear and connected, then it is not necessary to enlarge the field. Rosenlicht proved in [9] that in this case $G(K)$ is already Zariski dense.

Proposition 8.5. Let $G$ be an algebraic group defined over some number field $K$. Then there exists a finite field extension $L / K$ such that $G(L)$ contains a Zariski dense S-integral subset where $S$ denotes the set of all non-archimedean places of $L$.

Proof. Let $Z$ denote the center of $G$. By the theorem of Chevalley there is a unique maximal linear connected subgroup $H$ of $Z$ and furthermore the quotient $Z / H$ is an abelian variety. As the center of $G, Z$ is invariant under the action of the Galois group of $K$ and therefore $Z$ is defined over $K$. Similarily uniqueness of the maximal linear subgroup $H$ implies that $H$ is defined over $K$.

The quotient group $G / Z$ is linear, because the center $Z$ is the kernel of the adjoint representation of $G$. Over the algebraically closed field $\bar{K}$ every connected linear algebraic group is generated by its onedimensional connected algebraic subgroups. Moreover, every linear connected one-dimensional $\bar{K}$-group is isomorphic to either the additive group $G_{a}$ or the multiplicative group $G_{m}$. Hence there is a finite field extension $K^{\prime} / K$ such that $G / Z$ is generated by one-dimensional subgroups defined over $K^{\prime}$ each isomorphic to $G_{a}$ or $G_{m}$ over $K^{\prime}$. Therefore there is a dominant $K^{\prime}$-morphism from some $G_{a}^{n} \times G_{m}^{l}$ to $G / Z$. This implies that there is a Zariski dense integral subset $E$ in $G / Z$ (see 3.2.(6)). Similarily, we may assume that the connected linear group $H$ contains a Zariski dense integral subset $E_{H}$. By a further field extension $K^{\prime \prime} / K^{\prime}$ we may assume that both $G$ and $Z / H$ admit Zariski dense sets of $K^{\prime \prime}$-rational points (proposition 8.3). Now the projection $\tau: G / H \rightarrow G / Z$ is a proper morphism (because $Z / H$ is an abelian variety); hence $E^{\prime}=\tau^{-1}(E) \cap(G / H)\left(K^{\prime \prime}\right)$ is an integral subset of $G / H$. Moreover $E^{\prime}$ is Zariski dense in $G / H$, because $(Z / H)\left(K^{\prime \prime}\right)$ is Zariski dense in $Z / H$ and $E$ is Zariski dense in $G / H$. By definition, the algebraic group $H$ is connected, commutative and linear and therefore "special" in the sense of Serre ([10], proposition 14). For this reason, the quotient map $G \rightarrow G / H$ is a Zariski locally trivial fiber bundle and we may deduce from corollary 8.2 that there is an integral subset $E^{\prime \prime}$ of $G\left(K^{\prime \prime}\right)$ which surjects onto $E^{\prime}$. Finally we take the image of $E^{\prime \prime} \times E_{H}$ under the morphism $G \times H \rightarrow G$ given by group multiplication in order to get a Zariski dense integral subset of $G$. 


\section{RAMIFIED COVERINGS OVER SEMI-ABELIAN VARIETIES}

Here we prove theorem 1.5 .

Proof. First we observe that $\pi: X \rightarrow A$ fibers through the quasiAlbanese variety of $X$. Since $X$ is defined over $K$, so is its quasiAlbanese.

A semi-abelian variety admits only countably many semi-abelian subvarieties. As a consequence, if a semi-abelian variety is defined over $K$, then every semi-abelian subvariety is defined over some finite field extension of $K$. It follows that quotient of the given semi-abelian variety by this semi-abelian subvariety is likewise defined over $K$.

Applied to the quasi-Albanese $Q$ of $X$ and in consideration of the morphism from $Q$ to $A$ induced by $\pi: X \rightarrow A$ these arguments show that there is a finite extension $K_{0}$ of $K$ such that $A$ is defined over $K_{0}$.

By [8] there is a semi-abelian variety $B$, a morphism $\phi: B \rightarrow X$ and a holomorphic map $F: \mathbb{C} \rightarrow B$ such that $f=\phi \circ F$ and such that $\phi(B)$ equals the Zariski closure of $f(\mathbb{C})$ in $X$. Furthermore we may assume that the induced map $\pi \circ \phi: B \rightarrow A$ is finite. Then the above arguments on semi-abelian subvarieties imply that $B$ is defined over some finite field extension $K_{1}$ of $K_{0}$.

Now $\pi \circ \phi: B \rightarrow A$ is a morphism between two semi-abelian varieties which are both defined over $K_{1}$. It follows that there is an element $a \in A(\mathbb{C})$ and a morphism of $K_{1}$-group varieties $\zeta: B \rightarrow A$ such that $\pi \circ \phi$ and $\zeta$ differ by the map $\tau_{a}$ given by translation by $a$.

We regard the space of all morphisms $\psi$ from $B$ to $X$ with the property that there is an element $p \in A$ such that $\pi \circ \psi=\tau_{p} \circ \zeta$.

This is an algebraic variety which contains a $\mathbb{C}$-rational point. Thus it also contains a $\overline{\mathbb{Q}}$-rational point and consequently it contains a rational point for some finite field extension $K_{2}$ of $K_{1}$.

This yields a morphism $\psi: B \rightarrow X$ defined over $K_{2}$ whose image $\psi(B)$ has the same dimension as $\phi(B)$.

Next we chose a finite field extension $K_{3} / K_{2}$ such that $B\left(K_{3}\right)$ admits a Zariski dense integral subset $E$. (This is possible due to proposition 8.5.) Then $R=\phi(E)$ is a integral subset of $X\left(K_{3}\right)$ whose Zariski closure has the same dimension as the Zariski closure of $f(\mathbb{C})$.

\section{REFERENCES}

[1] Borel, A.: Some finiteness properties of adele groups over number fields. Publ. IHES 16, 5-30, (1963)

[2] Borel, A.: Linear Algebraic Groups. Second Edition. GTM 126. Springer 1991. 
[3] Grauert, H.: Holomorphe Funktionen mit Werten in komplexen Lieschen Gruppen. Math. Ann.133 , 450-472. (1957)

[4] Jouanolou, J.P.: Une suite exacte de Mayer-Vietoris en $K$-théorie algébrique. in: Algebraic $K$-theory I: Higher $K$-theories. Proceedings Conf. Seattle. Springer LN 341. 1973.

[5] Lang, S.: Fundamentals of Diophantine Geometry. Springer 1983.

[6] Nagata, M.: Imbedding of an abstract variety in a complete variety. J. Math. Kyoto Univ. 2 ,1-10. (1962)

[7] Noguchi, J.; Winkelmann, J.: Holomorphic Curves and Integral Points off Divisors. Math. Z. 239 , 593-610 (2002)

[8] Noguchi, J.; Winkelmann, J.; Yamanoi, K.: Degenerate Holomorphic Curves.

[9] Rosenlicht, M.: Some rationality questions on algebraic groups. Annali, 43, 25-50 (1957)

[10] Serre, J.P.: Espaces fibrés algèbriques. In: Séminaire Chevalley. 2ieme année. 1958. Anneaux de Chow et Applications.

[11] Silverman: Arithmetic of elliptic curves. GTM 106. Springer 1992.

[12] Vojta, P.: Diophantine Approximations and Value Distribution Theory. Springer LN 1239.

[13] Winkelmann, J.: Non-degenerate holomorphic maps and sets. Math. Z.249, 783-795 (2005)

Jörg Winkelmann, Mathematisches Institut, Universität Bayreuth, Universitätsstrasse 30, D-95447 Bayreuth, Germany,

E-mail address: jwinkel@member.ams.org

Webpage: http://btm8x5.mat.uni-bayreuth.de/ winkelmann/ 